\title{
Astronomy for international development
}

\author{
George Miley \\ Leiden Observatory \\ PO Box 9513, NL 2300 RA Leiden, The Netherland \\ email: miley@strw.leidenuniv.nl
}

\begin{abstract}
This review deals with the importance of astronomy for international development a nd capacity building. I shall summarize the present global state of astronomical research and education and describe current global astronomy education and development programs being carried out by the IAU and others. A decadal strategic plan will be described that envisages a substantial increase in IAU education and development activities during the next decade.
\end{abstract}

Keywords. Astronomy, International development, capacity building

\section{Introduction}

The International Astronomical Union regards furthering the exploitation of astronomy for sustainable global development as an important part of its mission. During this talk I shall first discuss the unique rôle of astronomy for international development and capacity building. After summarizing the present global state of the astronomical research and education and I shall describe some astronomy education and development programs that are currently being carried out by the IAU and others. Finally, I shall outline of an ambitious strategic plan that is being developed by the IAU. This plan envisages a substantial increase in IAU education and development activities during the next decade.

\section{Relevance of Astronomy for development}

From the dawn of history, astronomy has been an important factor in human development. The beauty and regularity of the sky has been a source of wonderment and the ability to predict the motions of the Sun and stars were decisive factors in the emergence of agriculture and navigation in early civilizations. A yearning for knowledge about our roots has resulted in a deep curiosity about the origin and history of the Sun and Moon, the stars and galaxies and the Universe itself.Because it embodies a unique combination of science, technology and culture, astronomy continues to play an important rôle in modern society. On the one hand astronomy has driven developments in several areas of advanced technology and on the other hand, the astronomer is the ultimate historian who delves much deeper into the past than conventional historians. There are several reasons why astronomy plays a special rôle in furthering the advancement of science and technology and imbuing students with useful skills. The Universe provides a laboratory for studying extreme conditions that are inaccessible on Earth. Stars and galaxies are environments that have produced the chemical elements around us and formed organic molecules, the building blocks of life. During the last century astronomical studies have led to new discoveries in physics, chemistry and biology and to the creation of the new sciences of astrophysics, astrochemistry and astrobiology. Because of its mathematical basis, astronomy is also an excellent tool for teaching mathematics. 
Astronomy has been an important driver for the development of advanced technology, such as the most sensitive detectors of light and radio waves and the fastest computers. The need to study the faintest objects possible requires sophisticated electronics and extreme-precision adaptive optics as well as state of the art engineering. Modern optical and radio telescopes are among the most advanced machines ever built and are outstanding educational vehicles for becoming familiar with the latest complex technology. Astronomy also contributes substantially to modern culture and is relevant to several topical issues of present-day society. Because radiation from the distant Universe takes so long to reach the Earth, the astronomer can probe deeply into our past. Large telescopes operating throughout the electromagnetic spectrum are time machines that routinely provide pictures and other information about of the observable Universe close to its birth, 13.7 billion years ago. Unravelling the history of the Universe has been a crowning achievement of humankind during the last half century. One of the most important societal functions of modern astronomy is as a tool for education in the broadest sense. Because it is one of the most approachable of sciences that consistently fascinates young people, astronomy is an excellent vehicle for introducing science and technology to children. The accessibility of the sky, the beauty of cosmic objects and the immensity of the Universe are inspirational and provide a perspective that encourages internationalism and tolerance. The excitement of astronomy has stimulated large numbers of young people to choose a career in science and technology, thereby contributing to the knowledge economy of many countries. Astronomy can contribute at every stage of education and capacity building.

- Primary education (ages 4-10). The early formative years are crucial in the development of the human value system. At these ages children can readily appreciate and enjoy the beauty of astronomical objects and can learn to develop a 'feeling' for the vastness of the Universe. The sky and the Universe can excite young children and stimulate their imaginations. Exposure to inspirational astronomical themes can help broaden the minds and stimulate a world-view. Furthermore, astronomy is an excellent and exciting introduction to the scientific method and the concept that nature can be interrogated by rational means.

- Secondary education (ages 11-18). Astronomy is an outstanding medium for stimulating the interest of secondary school students in science and technology. The Universe and space travel are fascinating subjects in their own right. These topics can be integrated into physics, chemistry, biology and mathematics teaching and provide a link with technology and engineering studies. Recently, educational networks of telescopes have been developed that enable school children throughout the world to do astronomical observations by means of the Internet and introduce children to exciting scientific research.

- Tertiary education and research training. The link with astronomy is a frequent reason for young people to choose to study the physical sciences at University and the study of astronomy provides an excellent preparation for many careers in technology and management. Astronomy deals with material, which is much denser and much sparser than anything that can be produced on Earth. Analysing phenomena under the extreme conditions that are present in astrophysical objects develops problem solving abilities. Furthermore, modern astronomical research is often carried out in international collaborative teams, which by necessity develops managerial and people skills.

- Research capabilities and infrastructures. Much modern astronomical research requires facilities that are too expensive even for individual developed countries to build and operate. The realization of such facilities has frequently necessitated large international collaborations. Nevertheless, many of the largest astronomical telescopes and 
satellites and their archival treasures can be used by astronomers throughout the world, no matter where they are based, providing an easy and relatively inexpensive entry for developing countries into inspirational and visible world-class international research.

- Public outreach. Astronomy is the most approachable of all sciences for the general public. Compare the relative attention that astronomy receives in the newspapers and other media of most countries with that devoted to most other sciences. Everybody can gaze at the sky and appreciate its beauty. The evocative images produced by modern telescopes fascinate, whereas stories about exotic cosmic objects and the evolution and origin of our Universe can inspire, entertain and stretch the imagination. Information about the state of the Universe in the distant past has deep implications about the roots and future of our species. Astronomy provides an ideal introduction for teenagers to the creative excitement of the exact sciences and frequently stimulates students to embark on a scientific career. The adventure of astronomy is a popular ingredient of adult education programs.

I mention South Africa as an excellent example and rôle model of a nation that is exploiting astronomy to further its development. See the contribution from Patricia Whitelock in this volume (page 590).

\section{Present state of World Astronomy development}

The present state of astronomical development, the degree of primary and secondary and tertiary education and the GDP of each country are all factors that need to be taken account in planning and optimising future strategy for astronomy development.

\subsection{Research}

An overview of the state of astronomy development was given by Hearnshaw (2007), who classified countries into various groups, using the GDP as a classification parameter. Here I shall use data supplied by Hearnshaw (2008) and adopt a slightly modified classification scheme, based purely on the state of astronomy in the countries. For the purposes of discussing world astronomical development, it is convenient1 to divide countries into the following groups:

Group 1A. 'Developed astronomy research countries A'. These are IAU member states with $>4$ IAU members per million population, indicative of a thriving astronomy research community.

Group 1B. 'Developed astronomy research countries B'. These are IAU member states that participate in or host front-line astronomy research facilities, but that have less than 4 members per million population.

Group 2. 'Emerging astronomy research countries'. These are IAU member states with between 0.5 and 4 IAU members per million population, that do not participate in front-line astronomy research facilities. They are targets for stimulating growth of their astronomical research.

Group 3. 'Developing astronomy research countries'. These are countries that do not adhere to the IAU, but have at least one individual IAU member, indicative of limited involvement in astronomical research. They are targets for stimulating growth of their astronomical research.

Group 4. 'Potential developing astronomy research countries'. These are countries with well developed tertiary education that neither adhere to the IAU nor contain individual IAU members. They are targets for stimulating the establishment of astronomy-oriented research groups. 


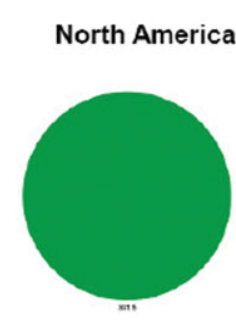

2

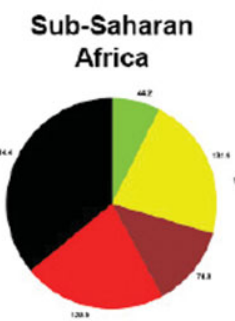

26

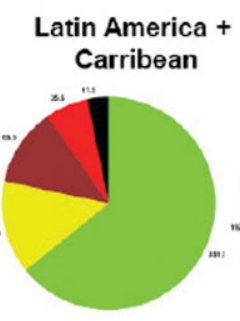

23

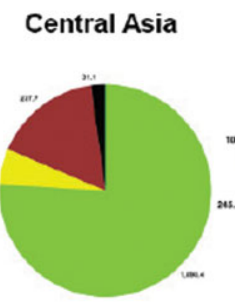

15

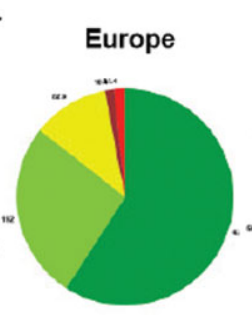

45

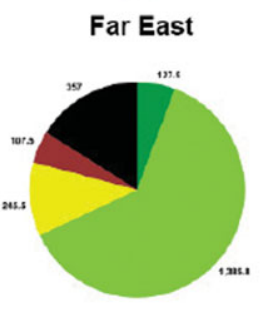

15

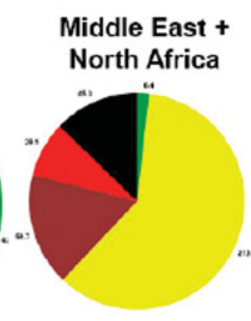

20

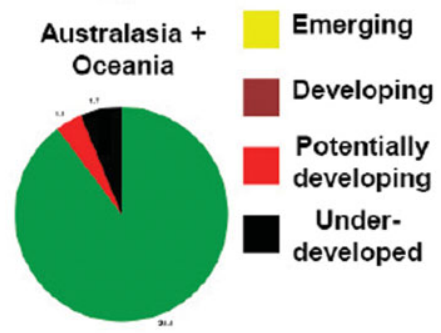

7

Figure 1. Astronomy research development by region. Population in millions that inhabit countries at various stages of development in different regions of the world. The plots were compiled on the basis of data from Hearnshaw (2008, private communication). The numbers of countries included in each region are indicated in blue.

Group 5. 'Underdeveloped astronomy countries'. These are countries that do not adhere to the IAU or contain individual IAU members whose tertiary education is only weakly developed. They are targets for stimulating the dissemination of astronomy education within their schools.

A summary of the present state of astronomical development in 152 countries as a function of their region is given in Figure 1. For each region the number of countries and the number of inhabitants that fall into each of the above classifications are shown.

A number of conclusions follow from these statistics. About two thirds of the world's population inhabits Group 1 countries that are developed in astronomical research. However, many of the developed astronomy countries in Group 1B have large populations and within these countries there are often substantial regional variations in the degree of astronomy development. There is considerable disparity from region to region. The region that has the largest populations in the least developed astronomical groups is Sub-Saharan Africa As to be expected, there is a strong correlation between astronomical development and gross domestic product (GDP), with poorer countries generally being less developed in astronomy.

\subsection{Education}

The state of the educational infrastructure must be an important factor in determining the detailed strategy for astronomy development, particularly in the areas of school education and public outreach. The global distribution of educational index is illustrated in Figure 2.

Since not all UN member states chose provide the necessary statistics, the data is not complete. Nevertheless, they provide a useful basis for planning future initiatives for programs directed at stimulating astronomy in primary and secondary and tertiary education. Countries can be divided into three broad categories based on their Education Index: high, medium, and low. As is the case with astronomy research development, 


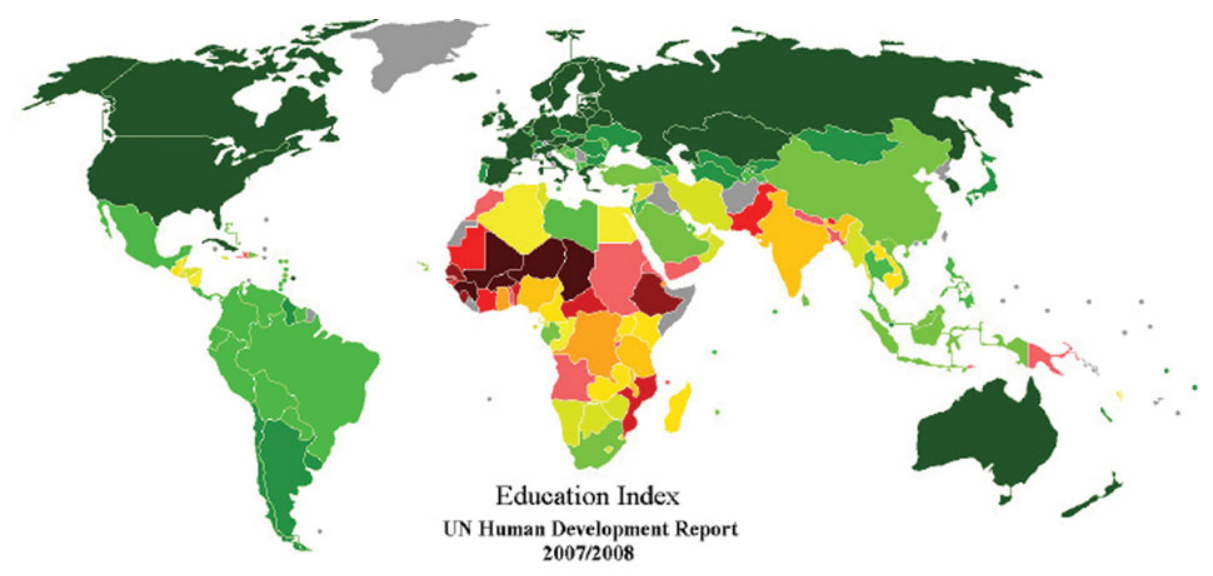

\begin{tabular}{lcc} 
High & \multicolumn{1}{l}{ Lew } \\
$\square 0.950$ and over & $0.750-0.799$ & $\square .450-0.499$ \\
$\square 0.900-0.949$ & $0.700-0.749$ & $0.400-0.449$ \\
$\square .850-0.899$ & $0.650-0.699$ & $\square 0.350-0.399$ \\
$0.800-0.849$ & $0.600-0.649$ & $\square$ under 0.350 \\
& $0.550-0.599$ & not available \\
& $0.500-0.549$ &
\end{tabular}

Figure 2. Global distribution of educational index. The educational index is defined by $E=(2 / 3) \times L+(1 / 3) \times C$, where $L$ is the literacy rate and $C$ is the combined gross school enrolment ratio. This is taken from the 2007/2008 edition of the UN Human Development Report (2007).

Sub-Saharan Africa has the largest number of least developed countries as measured by their educational index.

\section{Present global Astronomy development activities}

The IAU presently conducts a range of activities directed towards education and development, with emphasis on universities and research. Several complementary programs have recently been initiated by IAU members for exploiting astronomy in primary and secondary education. The Union presently works to promote astronomical education, research and public outreach through two commissions of its members. Commission 46 is concerned with 'Astronomy Education and Development' and Commission 55 is devoted to 'Communicating Astronomy with the Public'. The IAU Executive Committee performs an oversight rôle of development activities and, with the assignment of the portfolio of Development and Education to a Vice President, is playing an active rôle in the development of overall strategy. Until now, the coordination and implementation of all IAU activities pertaining to development and education has been carried out on a purely voluntary basis. As part of Commission 46, four main 'program groups' are involved with furthering astronomy in the developing countries. These program groups are the 'World Wide Development of Astronomy' (WWDA), 'Teaching for Astronomy Development' (TAD), the 'International Schools for Young Astronomers' (ISYA) and 'Exchange of Astronomers'. The present annual budget for all these activities is $\sim \mathrm{CHF} 120,000$, about 
Table 1. Present global activities for astronomy development.

\begin{tabular}{|c|c|c|c|c|}
\hline Public outreach & $\begin{array}{l}\text { Primary } \\
\text { education }\end{array}$ & $\begin{array}{l}\text { Secondary } \\
\text { education }\end{array}$ & $\begin{array}{l}\text { Tertiary } \\
\text { education }\end{array}$ & Research capability \\
\hline Japan: Tripod/ODA & UNAWE & $\begin{array}{l}\text { Hands-on Universe (HOU) } \\
\text { Las Cummbres/ } \\
\text { Faulkes Telescope } \\
\text { Japan: Tripod/ODA }\end{array}$ & $\begin{array}{l}\text { Comm. } 46 \text { TAD } \\
\text { Comm. } 46 \text { ISYA }\end{array}$ & $\begin{array}{l}\text { Comm. } 46 \text { WWDA } \\
\text { Comm. } 46 \text { TAD } \\
\text { Comm. } 46 \text { ISYA } \\
\text { Comm. } 46 \text { EA }\end{array}$ \\
\hline
\end{tabular}

Note: Shown here are programs conducted by the IAU and programs that are complementary to IAU activities in italics.

$10 \%$ of the total annual expenditure of the IAU. Some of these programmes will be described in detail elsewhere in this symposium. Specific IAU activities have until now been understandably biased towards developing tertiary education and building up research capabilities in astronomy. However, recently some programs have been initiated, outside the direct auspices of the IAU for stimulating astronomical education at the primary and secondary levels. These programs complement present IAU activities. A list of relevant IAU and complementary activities is given in Table 1. Taken together, they comprise a suite of activities that actively stimulate all elements of global astronomy, from the education of very young children to building up research and public understanding through the donation of small telescopes and planetaria. Global development at all levels is being emphasized in the activities of the International Year of Astronomy in 2009, an initiative of the IAU.

\subsection{Universe Awareness.}

Inspiring very young disadvantaged children Here I shall briefly mention Universe Awareness. This is a specific case of an astronomy outreach activity with both social and educational goals and one that I initiated and am closely involved with.

Universe Awareness (UNAWE) is an international outreach activity whose goal is to inspire young disadvantaged children with the beauty and grandeur of the universe. UNAWE exploits the inspirational aspects of modern astronomy to broaden children's minds, awaken their imagination and curiosity in science and stimulate global citizenship and tolerance. Games, songs, hands-on activities, cartoons and live internet exchanges are devised in partnership with UNAWE communities throughout the world for children from the age of 4 upwards. UNAWE enables the exchange of ideas and materials through networking and interdisciplinary workshops. The program has been adopted as a global cornerstone program of the International Year of Astronomy in 2009. UNAWE is now active in more than 21 countries in Europe and in the developing world, with a team of more than 100 dedicated volunteers $\dagger$

\section{The IAU decadal plan 2010-2020}

As Vice President with responsibility for Development and Education I am coordinating the development of an IAU strategic plan for astronomy development over the next decade. We finalised it at the IAU General Assembly in August 2009. The IAU intends to expand its rôle in furthering the use of astronomy at all levels in developing countries, working closely with relevant external organizations and using the International Year of Astronomy in 2009 as a springboard. The vast reservoir of talent presently active in astronomy throughout the world will be exploited and mobilized to further sustainable global development.

$\dagger$ UNAWE is described in more detail by Carolina Ödman in this volume, see page 650 . 
The long-term vision of the IAU is that:

- All countries will participate at some level in international astronomical research;

- All children throughout the world will be exposed to knowledge about astronomy and the Universe.

Goals for the next decade are:

- Raising the level of astronomy development in as many countries as possible, so as to maximize the size of population reached;

- Working to include aspects of astronomy as aids to the primary and secondary education of as many children as possible.

To achieve these goals existing efforts will be intensified, new programs will be initiated and the IAU development activities will be incorporated into a more professional organizational structure.

Ingredients of the strategy include the following:

- An integrated strategic approach involving primary, secondary, tertiary and research education and public outreach. The strategy will be based on the future potential for astronomy research and education in each country, using objective data, augmented by advice from experts in the region. Because of its relative underdevelopment, Sub-Saharan Africa is a region that will receive special attention.

- Enlarging the number of active volunteers. Present activities depend entirely on volunteers, both for their coordination and implementation. The IAU aims to enlarge the number of volunteer-experts by recruiting more members and augmenting the pool of volunteers by doctoral and postdoctoral trainees and talented non-member experts on pre-tertiary education and outreach.

- Initiation of new programs. The IAU will begin several new programs to stimulate astronomy development.

- An endowed lectureship program will provide semi-popular lectures on inspirational topics in modern astrophysics for high-school students and the general public in developing countries.

○ An institute twinning scheme will encourage developed astronomy institutes to provide long-term guidance and advice to university departments in developing countries interested in building up an astronomy research capability.

- Creation of a Global Development Office. Mobilizing a larger number of volunteers and implementing new programs cannot be achieved, without some professional coordination. A crucial step is the creation of a small IAU Global Development Office, led by an IAU Director of Development and Education.

- Increasing regional involvement. An important component of the plan is the adoption of a 'bottom-up' approach for astronomy development, with a substantial degree of decentralization. This will involve the appointment of regional development coordinators and the designation of regional 'institute nodes'. The regional coordinators will coordinate development efforts throughout their geographical region.

- Global task forces. After the pool of volunteers has been increased, the global activities will be consolidated into three task forces to cover the various sectors of astronomy development.

- Evaluation and assessment will be an essential part of every component of the plan. The strategy will be implemented flexibly, taking account of available funding. The total annual cost of the plan is about $€ 1$ million an order of magnitude larger than the present cost of the IAU astronomical development program, but very small compared with the annual global expenditure on astronomical research.

Funding the plan will need an innovative approach and action on several fronts. First, an 'astronomy development levy' on the IAU dues of wealthy countries will be 


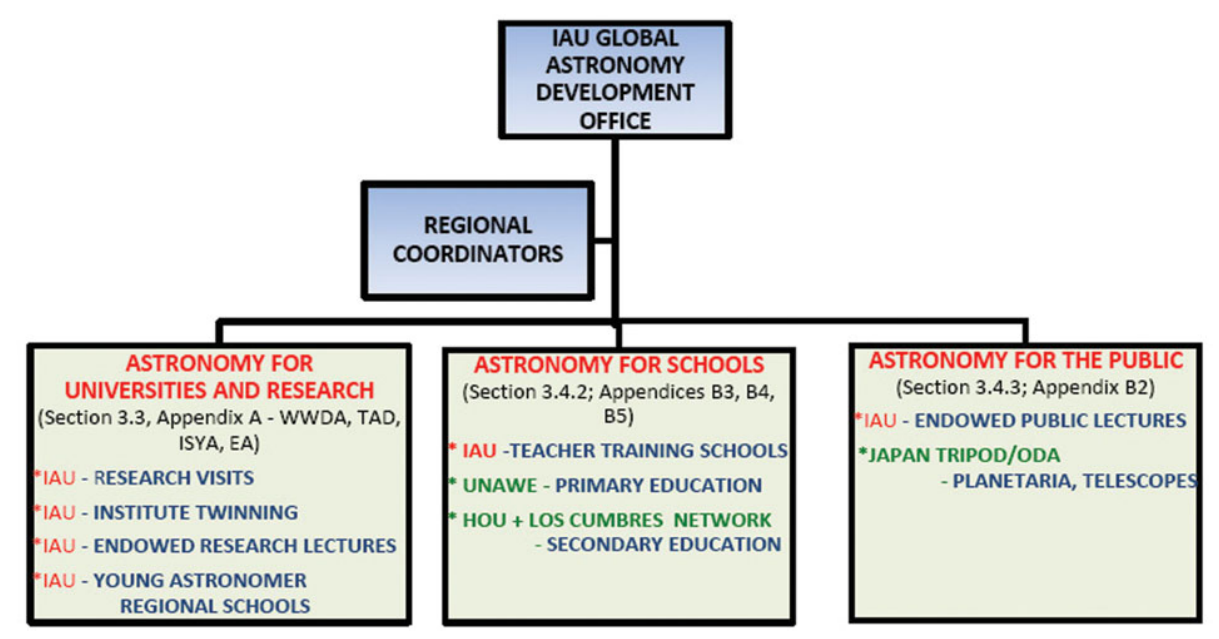

Figure 3. Future astronomy development activities to be coordinated by the three IAU task forces. Activities are shown in blue. IAU activities are prefixed by red and complementary programs are prefixed by green. Representatives of complementary programs will be included in the relevant task forces.

considered as a way of funding the IAU Global Development Office, an essential component of the plan. Secondly, vigorous attempts at external fund raising will be made, with approaches to international and regional aid agencies, national governments, industry and private foundations for support of various aspects of the plan. Thirdly, in-kind contributions from developed astronomical institutes and national astronomical societies will be sought. Fourthly, consideration will be given to adopting a target of at least $0.7 \%$ of the budgets of astronomical institutes and astronomical projects in rich countries for furthering astronomy education and research in developing countries. This would be in line with the well-established United Nations target for development aid.

\section{Acknowledgements}

I acknowledge useful discussions with John Hearnshaw about classification schemes and contributions from several colleagues in helping to develop the IAU Strategic Plan.

\section{References}

Hearnshaw, J. B. 2007, in J. B. Hearnshaw and P. Martinez (eds), Proc. Special Session 5, Astronomy for the Developing World, 26th IAU Gen. Assembly, (Cambridge: Cambridge University Pres), p. 9

Hearnshaw, J. B. 2008, Private communication

UN Human Development Report 2007, Climate Change and Human Development - Rising to the Challenge, United Nations Development Programme (New York: Palgrave Macmillan) 